\title{
Dermatoglifos en la criptorquidia infantil
}

\author{
Dres. N. Abodovsky ${ }^{*}$, X. Vivanco*, J. Herrera**, I. Avendaño*** y F. Pérez ${ }^{* * *}$
}

La criptorquidia es una anomalía que compromete aproximadamente al $10 \%$ de los recién nacidos. De éstos, el $3 \%$ aun presentan esta malformación al año de edad y alrededor del $1 \%$ la presentan todavía en la adolescencia, comprometiendo seriamente la fertilidad y la integridad psicológica del sujeto. Su tratamiento ha estado siempre en manos de cirujanos generales y urólogos, los cuales han considerado esta alteración exclusivamente desde el punto de vista mecánico y hormonal. La etiología genética no se ha considerado sino como un raro hallazgo. Ultimamente numerosos autores como Frazer, Ferguson Smith, Bergadá, etc., han mencionado en sus publicaciones a la criptorquídea asociada a patología genética como síndrome de Klinefelter, testículo rudimentario, pseudohermafroditismo masculino, etc.

*Depto. Endocrinología, Area Occidente. Hospital San Juan de Dios.

**Depto. Genética, Area Occidente, Hospital San Juan de Dios

***Depto. de Cenética, Area Oriente, Hospital (.) Mackenna.

****Técnica Laborista, Depto. Genética. Area Occidente, Hospital San Juan de IDios
Interesados en este problema, iniciamos, en 1968, el estudio genético de nuestros pacientes con criptorquidia, presentando en 1970, en el Congreso de Pediatría y Cirugía Infantil de Viña del Mar, un informe preliminar.

\section{MATERIAL. Y METODO}

Se analizan 88 pacientes tomados al azar entre los años 1968 y 1975, que consultaron a la Policlínica de Endocrinología y Genética del Hospital San Juan de Dios, por presentar criptorquidea. Su edad fluctuó entre los 3 y 15 años. En todos los pacientes se practicó una historia familiar dirigida, examen físico completo, dermatoglifos, cromatina sexual y cariograma en los casos indicados.

Se clasifició el material en dos grupos: criptorquidias unilaterales y criptorquidias bilaterales, que se comparan entre sí y con grupos controles. Los dermatoglifos se analizan separadamente, comparándose con controles chilenos del norte y sur del pais y un gitupo de tipo caucásico.

\section{RESULTADOS}

Las criptorquidias unilaterales aparecen en menor proporción que las bilaterales. En estas últimas encontramos 7 casos de cuadros genéticos (tabla 1). 
Tabla 1

Criptorquidias $\begin{cases}\text { Unilaterales } & 30 \\ \text { Bilaterales } & \text { Puras } \\ \text { Más cuadro genético } & 7\end{cases}$

Los cuadros genéticos están compuestos por disgenesias con una sola excepción (tabla 2).

Tabla 2

\begin{tabular}{|c|c|c|}
\hline \multirow{3}{*}{ Disgenesias testiculares } & S. de Klinefelter & 3 \\
\hline & & \\
\hline & $\begin{array}{l}\mathrm{S} . \mathrm{XY} / \mathrm{XO} \\
\text { Hermafroditismo }\end{array}$ & \\
\hline & S de Catlman & 1 \\
\hline
\end{tabular}

En los dermatoglifos se encuentra un recuento digital normal pero ligeramente alto en las criptorquidias uni o bilaterales, y bajo en el grupo genético. La proporción de las figuras digitales, el ángulo atd y el I P I se encuentran en las criptorquidias puras dentro de límites normales. En las genéticas, en cambio, se aprecia una inversión de algunos parámetros. El porcentaje de remolinos es bajo y el de asas es elevado, y el índice de Cumins es también bajo (tabla 3 ).

En la palma, el porcentaje de figuras en ei área interdigital 3 ( 9 en la nomenclatura moderna) es significativamente inferior a los controles en los casos puros, no así en las genéticas que en globo aparece elevado. Si discriminamos en estos enfermos separándolos por grupos, vemos que este porcentaje se mantiene bajo en los casos de Klinefelter y es elevado por el alto porcentaje que presentan en esta área las anomalías de Catlman y XO/XY (tabla 5).

Tabla

FIGURAS DIGITALES. RECUENTO DE DIGITOS IPI Y ANGLLO ATD EN CRIPTORQUIDIAS Y CONTROLES

\begin{tabular}{|c|c|c|c|c|c|c|}
\hline \multirow{4}{*}{ Figuras } & \multicolumn{3}{|c|}{ CRIPTORQUIDLAS } & \multicolumn{3}{|c|}{ CONTROLES } \\
\hline & \multirow{3}{*}{$\begin{array}{c}\text { Unilat. } \\
\qquad \%\end{array}$} & \multirow{3}{*}{$\begin{array}{l}\text { Bilat. } \\
\qquad \%\end{array}$} & \multirow{3}{*}{$\begin{array}{c}\text { Genét. } \\
\%\end{array}$} & \multicolumn{2}{|c|}{ Chilenos } & \multirow{3}{*}{$\begin{array}{c}\text { Exiranjeros } \\
\%\end{array}$} \\
\hline & & & & Norte & Sur & \\
\hline & & & & $\%$ & $\%$ & \\
\hline $\mathbf{R}$ & 38 & 39,2 & 27,1 & 41,8 & 27,6 & 28 \\
\hline $\mathrm{AC}$ & 51,6 & 51,6 & 65,7 & 48,5 & 58,9 & 61 \\
\hline AR & 2,3 & 4 & 2,9 & 5 & 3 & 6 \\
\hline Arcos & 7 & 5,2 & 4,3 & 4,6 & 10,4 & 4 \\
\hline Rec. Dig. & 165 & 166,4 & 130,3 & & 137,43 & 145 \\
\hline IPI & 13,2 & 13,5 & 11,8 & 13,7 & 11,6 & 11,7 \\
\hline atd & 44,6 & 44,4 & 52 & - & - & 42,6 \\
\hline
\end{tabular}

En el área 4 ( 7 de la nomenclatura moderna), las criptorquidias puras muestran porcentajes inferiores a los controles. Los genéticos, en cambio, muestran porcentajes normales (tabla 4).

Tabla 4

FIGURAS INTERDIGITALES TENARES E HIPOTENARES

\begin{tabular}{cccccc}
\hline Areas & $\begin{array}{c}\text { CRIPTORQuidias } \\
\text { Unilat. } \\
\%\end{array}$ & $\begin{array}{c}\text { Bilat. } \\
\%\end{array}$ & $\begin{array}{c}\text { Genét. } \\
\%\end{array}$ & $\begin{array}{c}\text { Controles } \\
\text { Chilenos } \\
\%\end{array}$ & $\begin{array}{c}\text { Extranjeros } \\
\%\end{array}$ \\
\hline 3 & 16,6 & 27 & 62,2 & 46,5 & 40 \\
4 & 30 & 31 & 45,8 & 50,9 & 49 \\
Tenar & 10 & 21 & 8,3 & 12 & 5 \\
Hipot. & 15 & 8 & 4,2 & 41,8 & 38 \\
\hline
\end{tabular}


Al discriminar los cuadros genéticos se ve que los Klinefelter tienen porcentajes similares a las criptorquidias, los hermafroditas valores normales, el $\mathrm{S}$. $\mathrm{XO} / \mathrm{XY}$ no tiene figuras $\mathrm{y}$ el S. de Catlman muy altos (tabla 5).

En el área hipotenar los valores son bajos, especialmente en los grupos genéticos. En el área tenar los porcentajes son superiores a los controles extranjeros, pero similares a los controles nacionales, con la sola excepción de los pacientes bilaterales que presentan un porcentaje igual al doble de los demás grupos.

\section{Tabla 5}

ANALISIS DE LOS CASOS GENETICOS EN GRUPOS SEPARADOS

\begin{tabular}{lcccc}
\hline & $\begin{array}{c}\text { Herma- } \\
\text { froditas } \\
\%\end{array}$ & $\begin{array}{c}\text { Kline- } \\
\text { feller } \\
\%\end{array}$ & $\begin{array}{c}X O / X Y \\
\%\end{array}$ & $\begin{array}{c}\text { Catiman } \\
\%\end{array}$ \\
\hline R & 10 & 46 & 0 & 30 \\
AC & 85 & 40 & 100 & 70 \\
AR & 0 & 7 & 0 & 0 \\
Arcos & 5 & 7 & 0 & 0 \\
IPI & 10,5 & 14 & 10 & 13 \\
atd & 49 & 47 & 42 & 71,5 \\
Area 3 & 50 & 0 & 100 & 100 \\
Area 4 & 50 & 33,3 & 0 & 100 \\
Tenar & 0 & 33,3 & 0 & 0 \\
Hipotenar & 0 & 16,6 & 0 & 0 \\
\hline
\end{tabular}

\section{COMENTARIO}

Del análisis de las figuras digitales llama la atención el bajo recuento de fajas digitales en los pacientes genéticos. $\mathrm{Al}$ parecer este hecho sería lógico, ya que el alto número de disgenesias que componen este grupo tendría un bajo porcentaje de remolinos y alto de asas. Esto es lo que ocurre en nuestros pacientes, pero no corresponde a la fórmula de nuestras disgenesias que tienen una proporción elevada de remolinos (tabla 5), sino a la bajísima proporción de remolinos que tienen los otros síndromes, como los hermafroditas y $\mathrm{XO} / \mathrm{XY}$. Creemos que eśto se debe a un fenómeno racial, ya que los chilenos tienden a presentar un alto porcentaje de remolinos. El ángulo atd que aparece más elevado que en los otros grupos, se debe a un fenómeno artificial, ya que es elevado por el síndrome de Catlman que tiene un gran desplazamiento de $\mathrm{t}$ y un ángulo atd de $71^{\circ}$.
Si no se excluye este resultado, el resto del grupo presenta un promedio normal.

Los dedos de los niños con criptorquidias, tanto uni como bilaterales, presentan valores muy dispersos, siguiendo una típica curva de Gauss, pero sus valores medios son normales.

En el análisis de las figuras palmares, nos sorprende la baja proporción que nuestros pacientes, especialmente los unilaterales, presentan en las áreas 3 e hipotenar. Por otra parte, los genéticos presentan porcentajes elevados en las áreas interdigitales y muy bajos en las áreas tenar e hipotenar. Al separar los pacientes genéticos en grupos homogéneos vemos que los sindromes de Klinefelter conservan proporciones semejantes a las criptorquidias puras, pero sus resultados son desviados por las fuertes alteraciones de los síndromes de Catlan y $\mathrm{XO} / \mathrm{XY}$. Los hermafroditas, por otra parte, mantienen porcentajes semejantes a los controles en las áreas interdigitales y no presentan figuras en las áreas tenar e hipotenar. Este hecho defraudaría un poco las expectativas, ya que es habitual esperar en los síndromes con alteración genética figuras en esta zona. Sin embargo, no podemos darle a estos resultados obtenidos en los pacientes genéticos, por su pequeño número, un carácter significativo, excepto los hallazgos clásicos de todos conocidos. Es lógico entonces que en este grupo se produzcan, a veces, grandes desviaciones de la media.

La cromatina sexual fue negativa en $\cdot 83$ pacientes. Sólo fue positiva en los 3 pacientes con síndrome de Klinefelter, y en los 2 pacientes con hermafroditismo verdadero. Este resultado es totalmente lógico y corresponde a los hallazgos más clásicos de estos síndromes.

\section{RESUMEN Y CONCLUSIONES}

El análisis de nuestro material nos permite anotar las siguientes conclusiones.

Las alteraciones que presentan los pacientes con criptorquidia en sus dermatoglifos, no son significativas, con la sola excepción del bajo porcentaje de figuras palmares. Pero son una gran ayuda para despistar los sindromes genéticos que acompañan a estas anomalías.

No se pueden seguir considerando las criptorquidias como un resultado único de un problema mexánico u hormonal, ya que los cuadros genéticos se presentan con una alta frecuencia $(10 \%)$.

La cromatina sexual constituye un excelente examen para descartar patología genética. y en conjunto con los dermatoglifos dan un alto porcentaje de seguridad a este diagnóstico. Nos permitimos hacer hincapié en la necesi- 
dad de incorporar estos exámenes a la rutina del estudio de las criptorquidias.

Los parientes de estos niños, creemos, necesitan un estudio especial, ya que sus dermatoglifos presentan variaciones que difieren bastante de los controles.

El cariograma sigue siendo un examen definitivo para confirmar diagnósticos sospechados por los 2 exámenes arriba mencionados, pero que dada su complejidad y alto costo sólo debe ser efectuado en casos totalmente justificados.

\section{SUMMARY}

Dermatoglyphic's alterations of criptorchidism are not significative, although they mean a great help in the diagnosis of genetic diseases.

Criptorchidism must not be considered only as the result of an hormonal or mechanic alteration because a high percentage $(10 \%)$ of genetic diseases are found in this anomaly.

We advice to practise both dermatoglyphic and sexual cromatine as any other routine control in criptorchidism.
We consider that patients' relatives should have an special investigation in attention of their dermatoglyphic alterations.

The kariotype remains being the definive examination in the diagnosis of this patients, but it must be done only in qualified cases.

\section{REFERENCIAS}

Atasu, $M$. et al. Lance, $2: 98,9-14$, jul. 73 .

David, T.J. Arch. Dis. Child, $48: 191-8$

Frazer, F.C. et al. Lancet, 1:682, 3-72.

Korf, B.R. et al. Lancet, 2:740, 29 Sep. 73.

Lacassie, Ives. Rev. Chil. Ped., 38:660-73, 1967 (I); 38: 730-37, 1967 (II)

Mateluna, E. J. Avendaño. Dermatoglifos en 2 ciudades del Norte de Chile. Antropología, vol. N, número 66-67.

Mateluna, E., X. Vicanco Dermatoglifos en 4 ciudades de la provincia de Chile. (en prensa)

Miller, J.R. J. Inv. Derm., 69:435-42, Jun. 73.

Penrose, L.S. Lancet, $1: 1239-42,2$ Jun. 73.

Trabajo entregado para su publicación en 1975. 